EPJ Web of Conferences 60, 14012 (2013)

DOI: $10.1051 /$ epjconf $/ 20136014012$

(C) Owned by the authors, published by EDP Sciences, 2013

\title{
Precision QCD in DIS at HERA
}

\author{
Eram Rizvi ${ }^{1, a}$ \\ ${ }^{1}$ Queen Mary, University of London, London, E1 4NS, UK
}

\begin{abstract}
The HERA ep collider experiments have measured the proton structure functions over a wide kinematic range. New data from the H1 experiment now extend the range to higher 4-momentum transfer $\left(\sqrt{Q^{2}}\right)$ over which a precision of $\sim 2 \%$ is achieved in the neutral current channel. A factor of two reduction in the systematic uncertainties over previous measurement is attained. The charged current structure function measurements are also significantly improved in precision. These data, when used in QCD analyses of the parton density functions (PDFs) reduce the PDF uncertainties particularly at high momentum fractions $x$ which is relevant to predictions for the LHC experiments as well as low energy neutrino scattering cross sections.
\end{abstract}

\section{Introduction}

Deep inelastic scattering data of lepton-nucleon interactions provide an excellent tool with which to measure the parton distribution functions [1]. The lepton provides a clean probe of the partonic content in charged current (CC) and neutral current (NC) interactions mediated by virtual $W$ and $Z / \gamma^{*}$ exchange respectively. In the regime of perturbative QCD $\left(Q^{2} \sim>1 \mathrm{GeV}^{2}\right)$ where the partonic degrees of freedom are the relevant means of describing the process, the measured cross sections can be related to the structure functions and the PDFs. Measurements from the HERA collider provide stringent constraints across a wide kinematic range in $x$ down to $10^{-4}$ and in $Q^{2}$ up to $10^{4} \mathrm{GeV}^{2}$. Data recently published by $\mathrm{H} 1$ [2] use the complete integrated luminosity from the HERA-II run to probe the region of $x>10^{-2}$ at high $Q^{2}$.

\section{Theory}

The differential cross section for $e^{ \pm} p$ scattering can be expressed in terms of generalised proton structure functions $\tilde{F}$ as $\frac{\mathrm{d}^{2} \sigma_{\mathrm{NC}}^{ \pm}}{\mathrm{d} x \mathrm{~d} Q^{2}}=\frac{2 \pi \alpha^{2}}{x Q^{4}}\left(Y_{+} \tilde{F}_{2}^{ \pm} \mp Y_{-} x \tilde{F}_{3}^{ \pm}-y^{2} \tilde{F}_{L}^{ \pm}\right)$, where $Y_{ \pm}=1 \pm(1-y)^{2}$ and $y=Q^{2} / s x$ with $\sqrt{s}$ the centreof-mass energy. The generalised structure functions, $\tilde{F}_{2,3}$, may be written as linear combinations of the proton structure functions $F_{2}, F_{2,3}^{\gamma Z}$, and $F_{2,3}^{Z}$ containing information on QCD parton dynamics. The structure function $F_{2}$ is associated to pure photon exchange terms, $F_{2,3}^{\gamma Z}$ correspond to photon- $Z$ interference terms and $F_{2,3}^{Z}$ describe the pure $Z$ exchange terms. The longitudinal structure function $\tilde{F}_{L}$ may be similarly decomposed, however this is an important contribution only at high $y$ and is expected to be negligible at large $x$ and $Q^{2}$. The linear combinations for $\tilde{F}_{2}$

\footnotetext{
ae-mail: e.rizvi@qmul.ac.uk
}

and $x \tilde{F}_{3}$ in $e^{ \pm} p$ scattering are given by

$$
\begin{gathered}
\tilde{F}_{2} \equiv F_{2}-v_{e} \frac{\kappa_{w} Q^{2}}{\left(Q^{2}+M_{Z}^{2}\right)} F_{2}^{\gamma Z}+\left(v_{e}^{2}+a_{e}^{2}\right)\left(\frac{\kappa_{w} Q^{2}}{Q^{2}+M_{Z}^{2}}\right)^{2} F_{2}^{Z} \\
x \tilde{F}_{3} \equiv-a_{e} \frac{\kappa_{w} Q^{2}}{\left(Q^{2}+M_{Z}^{2}\right)} x F_{3}^{\gamma Z}+\left(2 v_{e} a_{e}\right)\left(\frac{\kappa_{w} Q^{2}}{Q^{2}+M_{Z}^{2}}\right)^{2} x F_{3}^{Z},
\end{gathered}
$$

where $\kappa$ is the relative strength of $Z^{0}$ to photon exchange with $\kappa_{w}^{-1}=4 \frac{M_{W}^{2}}{M_{Z}^{2}}\left(1-\frac{M_{W}^{2}}{M_{Z}^{2}}\right)$ The quantities $v_{e}$ and $a_{e}$ are the vector and axial-vector couplings of the electron to the $Z$ boson. In the quark-parton model (QPM), the hadronic structure functions are related to linear combinations of sums and differences of the quark and antiquark momentum distributions $x q\left(x, Q^{2}\right)$ and $x \bar{q}\left(x, Q^{2}\right)$. The structure function $\tilde{F}_{2}$ is determined by the sum of quarks and anti-quark momentum distributions, whereas the structure function $x \tilde{F}_{3}$ is determined by the difference of quarks and anti-quark momentum distributions and is therefore sensitive to the valence quark distributions: $\left[F_{2}, F_{2}^{\gamma Z}, F_{2}^{Z}\right]=x \sum_{q}\left[e_{q}^{2}, 2 e_{q} v_{q}, v_{q}^{2}+a_{q}^{2}\right](q+\bar{q})$ and $\left[x F_{3}^{\gamma Z}, x F_{3}^{Z}\right]=2 x \sum_{q}\left[e_{q} a_{q}, v_{q} a_{q}\right](q-\bar{q})$. Here $v_{q}$ and $a_{q}$ are the vector and axial-vector couplings of the quarks to the $Z$ boson and $e_{q}$ is the charge of the quark of flavour $q$.

The reduced NC cross section is defined by

$$
\tilde{\sigma}_{\mathrm{NC}}^{ \pm}\left(x, Q^{2}\right) \equiv \frac{\mathrm{d}^{2} \sigma_{\mathrm{NC}}^{ \pm}}{\mathrm{d} x \mathrm{~d} Q^{2}} \frac{x Q^{4}}{2 \pi \alpha^{2}} \frac{1}{Y_{+}} \equiv\left(\tilde{F}_{2}^{ \pm} \mp \frac{Y_{-}}{Y_{+}} x \tilde{F}_{3}^{ \pm}-\frac{y^{2}}{Y_{+}} \tilde{F}_{L}^{ \pm}\right) .
$$

The differential CC cross section for $e^{ \pm} p$ scattering can be expressed as

$$
\frac{\mathrm{d}^{2} \sigma_{\mathrm{CC}}^{ \pm}}{\mathrm{d} x \mathrm{~d} Q^{2}}=\left(1 \pm P_{e}\right) \frac{G_{F}^{2}}{4 \pi x}\left[\frac{M_{W}^{2}}{M_{W}^{2}+Q^{2}}\right]^{2}\left(Y_{+} W_{2}^{ \pm} \mp Y_{-} x W_{3}^{ \pm}-y^{2} W_{L}^{ \pm}\right),
$$

where $G_{F}$ is the Fermi constant defined using the weak boson masses. Here $W_{2}^{ \pm}, x W_{3}^{ \pm}$and $W_{L}^{ \pm}$are the structure functions for $\mathrm{CC} e^{ \pm} p$ scattering. In the QPM $W_{L}^{ \pm} \equiv 0$, and the structure functions $W_{2}^{ \pm}$and $x W_{3}^{ \pm}$are expressed 
as the flavour dependent sum and difference of the quark and anti-quark momentum distributions. In the $\mathrm{CC}$ case only the positively charged quarks contribute to $W^{-}$mediated scattering and conversely only negatively charged quarks couple to the exchanged $W^{+}$boson, thus $W_{2}^{-}=$ $x(U+\bar{D}), \quad W_{2}^{+}=x(\bar{U}+D), x W_{3}^{-}=x(U-\bar{D}), \quad x W_{3}^{+}=$ $x(D-\bar{U})$, where, below the $b$ quark mass threshold $U=$ $u+c, \bar{U}=\bar{u}+\bar{c}, \quad D=d+s, \bar{D}=\bar{d}+\bar{s}$. Here $U$ represents the sum of up-type, and $D$ the sum of down-type quark densities, and $u, d, s, c$ represent quark densities of each flavour in the standard notation. The reduced CC cross section is then defined as

$$
\tilde{\sigma}_{\mathrm{CC}}\left(x, Q^{2}\right) \equiv \frac{4 \pi x}{G_{F}^{2}}\left[\frac{M_{W}^{2}+Q^{2}}{M_{W}^{2}}\right]^{2} \frac{\mathrm{d}^{2} \sigma_{\mathrm{CC}}}{\mathrm{d} x \mathrm{~d} Q^{2}} .
$$

\section{HERA DIS Data}

The HERA data is divided into two running periods, HERA-I in which $\sim 110 \mathrm{pb}^{-1}$ was collected, and HERAII in which about $330 \mathrm{pb}^{-1}$ was accumulated. Using the complete HERA-II data set the integrated luminosity is increased by a factor of three for $e^{+} p$ scattering and a factor of ten for $e^{-} p$ scattering data compared to HERA-I.

The NC reduced cross section measurements from $\mathrm{H} 1$ are shown in fig. 1(a) including measurements at low $Q^{2}$ shown as black solid points, and the new high $Q^{2}$ data shown in blue for $e^{-} p$ scattering and red for $e^{+} p$ scattering. For visualisation purposes the data in each $x$ bin are scaled by a factor as indicated on the figure. In the high $Q^{2}$ region an experimental precision of $\sim 2 \%$ is reached and is limited by the systematic uncertainties of the data. At higher $Q^{2}>500 \mathrm{GeV}^{2}$ the statistical uncertainties dominate the measurement precision. Compared to earlier preliminary measurements the $x$ range of the data is extended up to $x=0.65$.

The solid curve represents the next-to-leading order (NLO) H1PDF2012 QCD fit to the data which provides a good description of the data, and is discussed below. For $Q^{2}<\sim 1000 \mathrm{GeV}^{2}$ the cross sections are mostly sensitive to the $\tilde{F}_{2}$ structure function and the charge weighted sum of quarks and anti-quarks. The $Q^{2}$ dependence of the measurements at fixed $x$ show strong logarithmic scaling violations which are positive at low $x$ and negative at high $x$. These arise from higher order effects of gluon emission and allow constraints on the gluon density to be obtained.

The $\mathrm{H} 1$ measurements are shown in fig. 1(b) for the reduced CC cross section in $e^{+} p$ scattering and span the range in $Q^{2}$ from 300 to $15000 \mathrm{GeV}^{2}$. The experimental precision reaches $\sim 5 \%$ and is limited by the statistical uncertainty due to the supression from the weak $M_{W}$ propagator, similar to the NC $x \tilde{F}_{3}$ case. The H1PDF2012 fit is also shown and describes the measurements well. These data provide crucial flavour sensitivity through the different couplings to the $W$ in $e^{+} p$ and $e^{-} p$ scattering modes. At high $x$ the cross section is dominated by the $(1-y)^{2}(d+s)$ term in $e^{+} p$ scattering and is shown as the dashed curve. Since the $s$ quark PDF is small at high $x$, these cross sections provide a clean measure of the $d$ PDF.
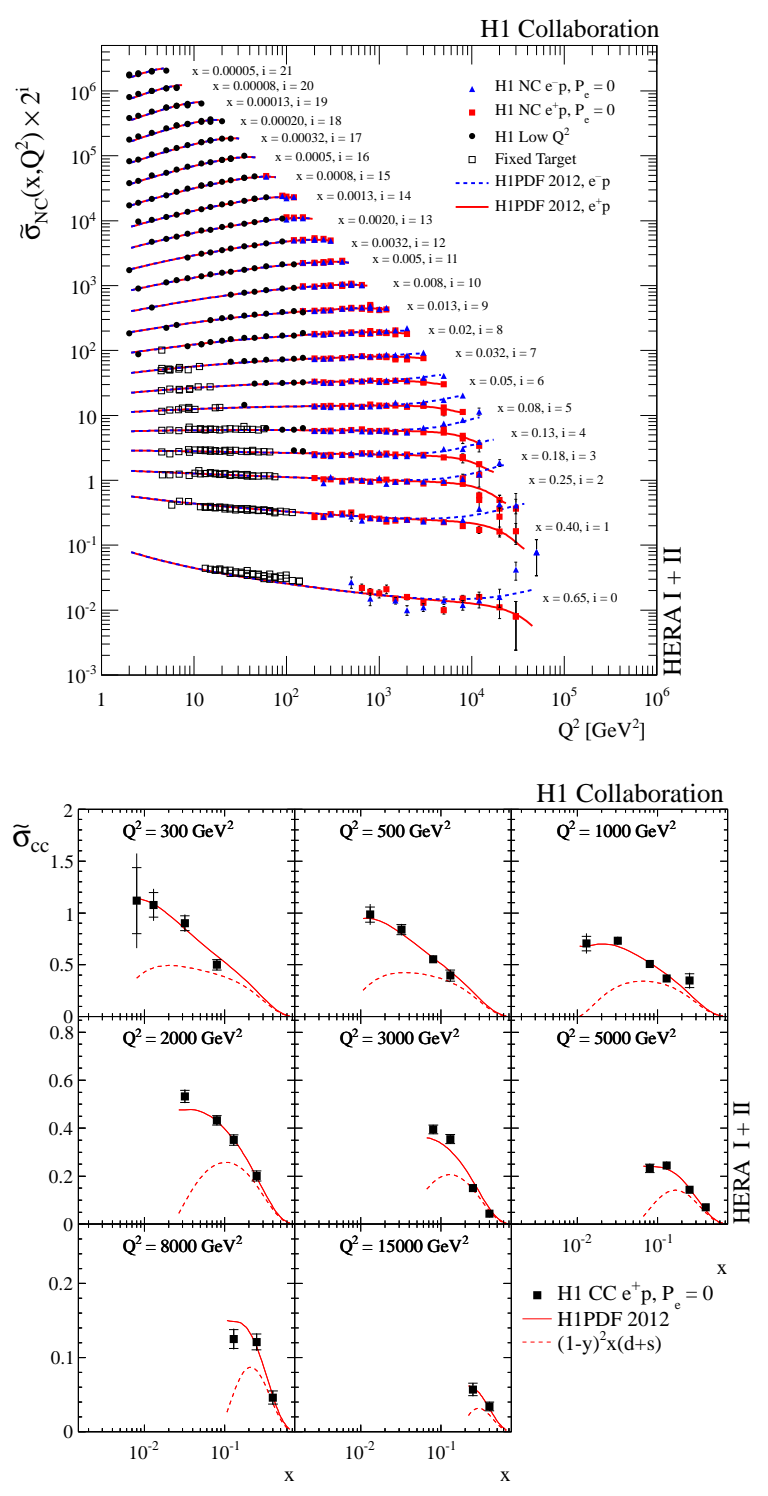

Figure 1. Measurements of the NC (left) and CC (right) scatterng cross sections from $\mathrm{H} 1$ using the complete HERA-II data set. The NC reduced cross section is shown for $e^{+} p$ and $e^{-} p$ scattering. The $\mathrm{CC}$ data are shown for $e^{+} p$ scattering, and both are compared to a NLO QCD fit.

In global analyses of DIS data [3-5] the constraints on the $d$ PDF at high $x$ are obtained from lower energy fixed target data, and deuteron targets which are subject to theoretical uncertainties arising from nuclear wave-functions and target mass effects (see for example the discussion in [1]). The HERA data are free from such uncertainties. In $e^{-} p$ scattering the $\mathrm{CC}$ reduced cross section is sensitive to the $u$ PDF at high $x$, although this is more precisely obtained from the NC $F_{2}$ contribution.

The $x \tilde{F}_{3}$ structure function becomes a significant contribution to the cross section for $Q^{2} \sim M_{Z}^{2}$ and causes an enhancement of the $e^{-} p$ scattering cross section and a suppression of the $e^{+} p$ measurements. This can be seen in all $x$ bins at high $Q^{2}$ in fig. 1(a). A direct measurement of $x \tilde{F}_{3}$ is obtained by subtracting the reduced cross section 


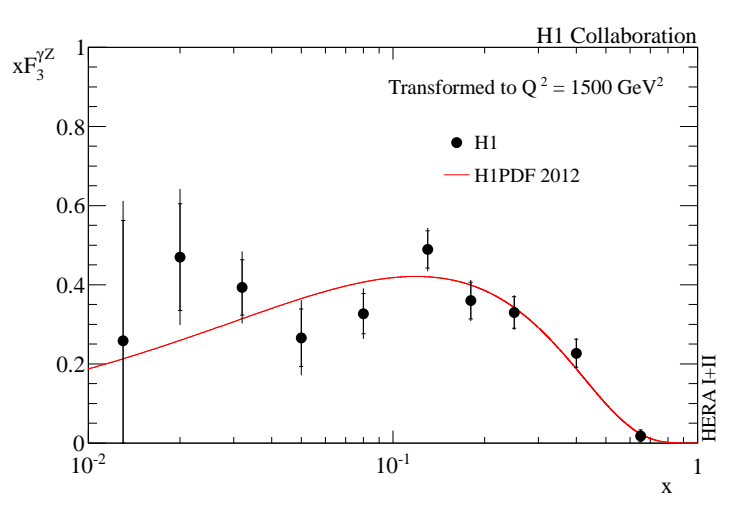

Figure 2. Measurements of $x \tilde{F}_{3}$ using the complete H1 data set. The data are compared to a NLO QCD fit.

measurements since $x \tilde{F}_{3}=\frac{Y_{+}}{2 Y_{-}}\left(\tilde{\sigma}_{\mathrm{CC}}^{-}-\tilde{\sigma}_{\mathrm{CC}}^{+}\right)$. The structure function is shown in fig. 2 compared to the H1PDF2012 fit. These data provide good sensitivity to the valence quark $u_{v}=u-\bar{u}$ and $d_{v}=d-\bar{d}$ densities.

\section{QCD Analysis}

The measurements have been used in a QCD analysis at NLO to extract the PDFs of the proton, termed the H1PDF2012 fit. The method follows the prescriptions and techniques used in the HERAPDF series of analyses [6]. The fit includes only H1 inclusive DIS data and therefore do not have issues related to the consistency of data sets from various experiments which often require special treatment in more global analyses [3-5]. The fitted data are restricted to the region $Q>3.5 \mathrm{GeV}^{2}$ to restrict the analysis to the perturbative regime. The RT generalised mass heavy flavour scheme is used in the fit [7]. The $\chi^{2}$ function takes into account the correlated experimental systematic uncertainties by introducing a nuisance parameter for each source which is varied within the fit.

The fitted PDFs are taken to be $x g, u_{v}, d_{v}, \bar{U}$, and $\bar{D}$ which are the combinations the HERA NC and CC data are most sensitive to. The parameterisations at the starting scale $Q_{0}^{2}=1.9 \mathrm{GeV}^{2}$ are chosen to be

$$
\begin{aligned}
x g(x) & =A_{g} x^{B_{g}}(1-x)^{C_{g}}-A_{g}^{\prime} x^{B_{g}^{\prime}}(1-x)^{C_{g^{\prime}}}, \\
x u_{v}(x) & =A_{u_{v}} x^{B_{u_{v}}}(1-x)^{C_{u_{v}}}\left(1+E_{u_{v}} x^{2}\right), \\
x d_{v}(x) & =A_{d_{v}} x^{B_{d_{v}}}(1-x)^{C_{d_{v}}} \\
x \bar{U}(x) & =A_{\bar{U}} x^{B_{\bar{U}}}(1-x)^{C_{\bar{U}}} \\
x \bar{D}(x) & =A_{\bar{D}} x^{B_{\bar{D}}}(1-x)^{C_{\bar{D}}} .
\end{aligned}
$$

where the normalistion parameters $A_{u_{v}}, A_{d_{v}}$ and $A_{g}$ are fixed by the valence quark sum rules, and the momentum sum rule. The parametric form for the gluon allows extra flexibility in the low $x$ region, and $C_{g^{\prime}}$ is set to 25 to suppress the negative contribution at high $x$. Relaxing the parameter $C_{g^{\prime}}$ does not cause significant changes to the fit results. The fraction of strange to light quark sea is set by $f_{s}=\bar{s} / \bar{D}=0.31$. The parameters controlling the low $x$ behaviour of the $\bar{U}$ and $\bar{D}$ are constrained through the relation $B_{\bar{U}}=B_{\bar{D}}$.

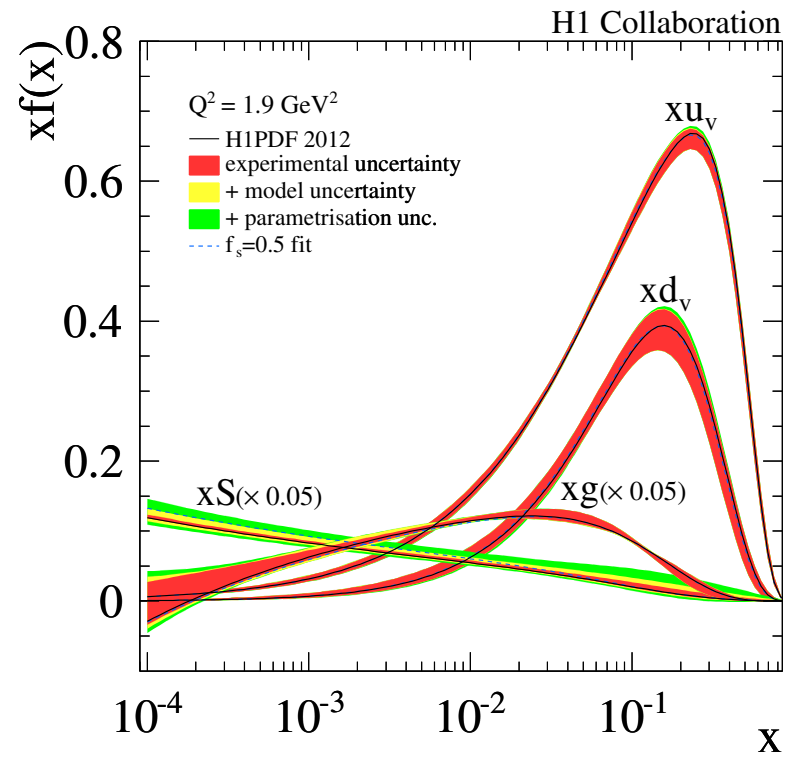

Figure 3. The PDFs determined in the H1PDF2012 NLO QCD fit at the starting scale $Q_{0}^{2}=1.9 \mathrm{GeV}^{2}$.

The number of additional parameters in the final polynomial terms is chosen according to the $\chi^{2}$ saturation technique: starting from a 10 parameter fit, all fits with one additional parameter are performed and the one with the smallest $\chi^{2}$ is chosen for a further iteration. This procedure is continued till the $\chi^{2}$ function no longer continues to significantly reduce. This is obtained with the 13 parameters above and is used for the central value of the fit. In order to estimate the uncertainty from parameterisation bias the envelope of all 14 parameter fits is used as well as the variation in the starting scale from 1.4 to $2.5 \mathrm{GeV}^{2}$.

Experimental uncertainties are evaluated from fits to 400 replica data sets in which the data points are randomly fluctuated according to their uncertainties. The RMS is used to define the experimental uncertainty band. Further theoretical uncertainties are estimated by varying the charm quark mass $(1.35-1.65 \mathrm{GeV})$ and bottom quark mass $(4.3-5.0 \mathrm{GeV})$, and the minimum $Q^{2}$ cut applied to the data from $\left(2.5-5.0 \mathrm{GeV}^{2}\right)$.

The fit results in a $\chi^{2}=412$ for 441 degrees of freedom. None of the 22 nuisance parameters are found to deviate significantly from zero. The results of the PDF fit are shown in fig. 3 for the individual PDFs at the starting scale $Q_{0}^{2}$. The uncertainty on the valence and gluon distributions are dominated by the experimental precision shown in red. For the sea quarks the uncertainty is mostly dominated by the parameterisation bias estimate, and to a lesser extent by the theoretical uncertainties of the fit.

In order to asses the influence of the new data, the fit is performed with and without the new $\mathrm{H} 1$ measurements. The PDF uncertainties are shown in fig 4 where the reduction in the error is visible as the difference between the red and green bands. The most significant reduction is in the $x D$ and $d_{V}$ at high $x$ arising from the more precise $\mathrm{CC} e^{+} p$ reduced cross section measurements. Sizeable reductions in the uncertainty are also visible for the $u_{v}$ at low $x$ which 
comes from improved high $x$ NC constraints propagated to low $x$ via the counting sum rules. The gluon uncertainty is also reduced at high $x$.

\section{Conclusions}

In summary the new data from the $\mathrm{H} 1$ experiment are able to improve our knowledge of the PDFs, particularly in the high $x$ region. These data allow the predictions for processes at the LHC to determined more accurately including novel phenomena at high $Q$ as well as Higgs production cross sections. Additionally in the neutrino sector, more accurate predictions are needed for low energy (and high $x$ ) neutrino-nucleon cross sections in the DIS regime which these data will help constrain.
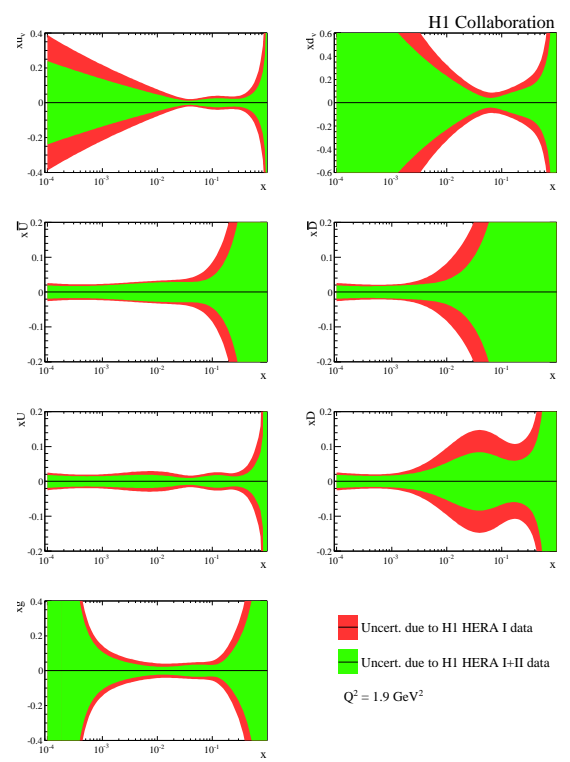

Figure 4. The PDF uncertainties determined in the H1PDF2012 NLO QCD fit obtained from two fits including and excluding the new $\mathrm{H} 1$ structure function measurements.

\section{References}

[1] E. Perez, E. Rizvi, Rep.Prog.Phys. 76, 046201 (2013), 1208. 1178

[2] F. Aaron et al. (H1 Collaboration), JHEP 1209, 061 (2012), 1206.7007

[3] A. Martin, W. Stirling, R. Thorne, G. Watt, Eur.Phys.J. C63, 189 (2009), 0901.0002

[4] R.D. Ball, V. Bertone, S. Carrazza, C.S. Deans, L. Del Debbio et al., Nucl.Phys. B867, 244 (2013), 1207.1303

[5] H.L. Lai, M. Guzzi, J. Huston, Z. Li, P.M. Nadolsky et al., Phys.Rev. D82, 074024 (2010), 1007. 2241

[6] F. Aaron et al. (H1 and ZEUS Collaboration), JHEP 1001, 109 (2010), 0911.0884

[7] R. Thorne, R. Roberts, Phys.Rev. D57, 6871 (1998), hep-ph/9709442 